\title{
Simultaneous voltammetric determination of heavy metals by use of crown ether-modified electrodes and chemometrics
}

\author{
Andreu González-Calabuig ${ }^{a}$, David Guerrero ${ }^{a}$, Núria Serrano ${ }^{b}$, Manel del Valle $^{a, *}$ \\ a Sensors\& Biosensors Group, Department of Chemistry, Universitat Autònoma de Barcelona, Edifici Cn, 08193 - Bellaterra \\ (Spain) \\ b Department of Analytical Chemistry, Faculty of Chemistry, Universitat de Barcelona, Martí i Franquès 1-11, 08028 - \\ Barcelona (Spain) \\ * e-mail: manel.delvalle@uab.es
}

Received: ((will be filled in by the editorial staff))

Accepted: ((will be filled in by the editorial staff))

\begin{abstract}
A three-sensor array consisting of a graphite-epoxy composite electrode (GEC), 4-carboxybenzo-18-crown-6-GEC and 4carboxybenzo-15-crown-5-GEC was employed for the simultaneous determination of $\mathrm{Cd}(\mathrm{II}), \mathrm{Pb}$ (II) and $\mathrm{Hg}$ (II) by differential pulse anodic stripping voltammetry (DPASV). Sensors were firstly studied for the determination of $\mathrm{Hg}$ (II); secondly, peak current responses confirmed that all sensors showed differentiated response for the three considered metals. A response model was developed to resolve mixtures of $\mathrm{Cd}(\mathrm{II}), \mathrm{Pb}(\mathrm{II})$ and $\mathrm{Hg}(\mathrm{II})$ at the $\mu \mathrm{g} \mathrm{\textrm {L } ^ { - 1 }}$ level; Discrete Wavelet Transform was selected as preprocessing tool and artificial neural network used for the modelling of the obtained responses.
\end{abstract}

Keywords: crown ether-modified sensors; electrochemical grafting; heavy metal; stripping voltammetry; artificial neural network

DOI: 10.1002/elan.((will be filled in by the editorial staff))

\section{Introduction}

The determination of heavy metal ions at trace levels is becoming more important day by day because of the health problems that they can cause in living systems, since they tend to bioaccumulate in their organisms and severe illnesses may be originated. Heavy metals become toxic when they are not metabolized by the body and accumulate in the soft tissues [1]. The main threats to human health from heavy metals are associated with exposure to lead, cadmium, mercury and arsenic. The general population is primarily exposed to mercury via food; fish is the major source of methyl mercury exposure, as also is relevant the use of dental amalgam. Related to cadmium exposure, cigarette smoking is the major source, whereas in nonsmokers, food is the most important source. Exposure to lead is mainly via air and food in approximately equal proportions, being children particularly vulnerable [2].

Atomic absorption spectrometry (AAS), inductively coupled plasma optical emission spectrometry (ICPOES), X-ray fluorescence (XRF), inductively coupled plasma mass spectrometry (ICP-MS), and stripping voltammetric techniques (anodic stripping voltammetry (ASV) and adsorptive stripping voltammetry (AdSV)) are some of the available techniques for the determination of heavy metals in natural samples. Particularly, stripping voltammetry techniques are the most suitable techniques for trace metals analysis in natural samples [3], due to their excellent detection limits, their sensitivity to the presence of different metal species, their capacity to multielement determination, and their relative low cost. Nevertheless, the performance of voltammetry is strongly influenced by the working electrode material. With the aim of developing alternative electrodes for classic mercury electrodes, chemically modified electrodes were considered for metal ion determination.

Thiol rich peptides and macrocyclic compounds, as crown ethers, can be employed as modifiers for metal determination. A simple and flexible approach to attach these compounds to an electrode surface is based on their immobilization on aryl diazonium salt monolayers 
anchored on the electrode surface, also referred in the literature as electrochemical grafting, which is a valuable alternative to the use of self-assembled monolayers (SAM) for forming stable complexing surfaces [4-7].

Particularly, crown ethers may act as a host, complexing a centre metal, and once introduced into the electrode enhance its selectivity and lower the metal detection limit. To achieve high degree of selectivity one may think to modify them to strongly bind certain metals allowing the complex formation by means of ion-dipole interaction with these metal ions [8]. So, the complexing ability and crown cavity size which is suitable for a particular metal ion have to be considered for its selective detection.

Although some studies devoted to the application of crown ether-modified electrodes for the individual determination of lead [9-11], mercury [12, 13], silver [14], thallium [15], palladium [16] and copper [17] can be found in the literature, related works entailing the simultaneous determination of different heavy metal ions are scarce [6].

Crown ether-modified electrodes not only can be used for metal determination as a single-electrode sensor but also in combination with others forming an electrode arrays with semi-selective ligands for the determination of several metal ions. Such a strategy relies on the modification of electrodes in the array with different complexing agents to provide a highly variate response [18]. The main advantage of a multi-sensor array over the classical one-electrode is that the information provided by the electrode array is significantly higher than that obtained from a single electrode.

In this work, a three-sensor array consisting of one graphite-epoxy composite electrode (GEC) and two GECs modified with 4-carboxybenzo-18-crown-6 (CB18-crown-6) and 4-carboxybenzo-15-crown-5 (CB-15crown-5) respectively, which were immobilized through aryl diazonium salt monolayers anchored to the electrode surface was firstly analytically studied for the determination of $\mathrm{Hg}$ (II) using voltammetric techniques. Subsequently, this three-sensor array was applied for the first time for the simultaneous determination of $\mathrm{Cd}(\mathrm{II}), \mathrm{Pb}$ (II) and $\mathrm{Hg}$ (II) ions in certified samples by voltammetric techniques. An artificial neural network model was proposed as a tool to maximize the information obtained from the voltammetric data.

\subsection{Chemicals}

Potassium ferricyanide $\mathrm{K}_{3}\left[\mathrm{Fe}(\mathrm{CN})_{6}\right]$, potassium ferrocyanide $\quad \mathrm{K}_{4}\left[\mathrm{Fe}(\mathrm{CN})_{6}\right], \quad$ 2-(N-morpholino)ethanesulfonic acid (MES), potassium dihydrogen phosphate, sodium monophosphate, methanol, perchloric acid, hydrochloride acid, $N$-hydroxysulfosuccinimide (sulfo-NHS), $\quad N$-(3-dimethylaminopropyl)- $N$ 'ethylcarbodiimide hydrochloride (EDC) and sodium nitrite were purchased from Sigma (St. Louis, MO, USA). 4-aminobenzoic acid (ABA) and DL-lysine monohydrochloride were provided by Acros (Geel, Belgium). 4-carboxybenzo-18-crown-6 with a purity of 99\% and 4-carboxybenzo-15-crown-5 with purity greater than $98 \%$ were provided by Acros and Sigma respectively. All other reagents used were from Merck (Darmstadt, Germany) and Fluka (Buchs, Switzerland). All reagents were of analytical grade. $\mathrm{Pb}(\mathrm{II}), \mathrm{Cd}(\mathrm{II})$ and $\mathrm{Hg}$ (II) stock solutions $10^{-2} \mathrm{~mol} \cdot \mathrm{L}^{-1}$ were prepared from $\mathrm{Pb}\left(\mathrm{NO}_{3}\right)_{2} \cdot 4 \mathrm{H}_{2} \mathrm{O}, \mathrm{Cd}\left(\mathrm{NO}_{3}\right)_{2} \cdot 4 \mathrm{H}_{2} \mathrm{O}$ and $\mathrm{Hg}\left(\mathrm{NO}_{3}\right)_{2} \cdot \mathrm{H}_{2} \mathrm{O}$ respectively and standardized complexometrically. $\mathrm{Pb}(\mathrm{II})$, $\mathrm{Cd}(\mathrm{II})$ and $\mathrm{Hg}$ (II) $1000 \mathrm{mg} \cdot \mathrm{L}^{-1}$ certified standard solutions were purchased from Fluka. $0.1 \mathrm{~mol} \cdot \mathrm{L}^{-1}$ acetic acid/acetate buffer solution ( $\mathrm{pH} 4.5$ ) was used for $\mathrm{pH}$ control. Ultrapure water from MilliQ System (Millipore, Billerica, MA, USA) was used in all experiments.

\subsection{Apparatus}

Voltammetric measurements were performed in an Autolab System PGSTAT 30 (EcoChemie, The Netherlands), in a multichannel configuration, using GPES Multichannel 4.7 software package (EcoChemie). The voltammetric cell was formed by one working graphite epoxy electrodes (GEC) and two working graphite epoxy electrodes modified with 4-carboxybenzo18-crown-6 (CB-18-crown-6) and 4-carboxybenzo-15crown-5 (CB-15-crown-5) respectively, a commercial platinum counter electrode (Model 52-67, Crison Instruments, Barcelona, Spain), and a double junction $\mathrm{Ag} / \mathrm{AgCl}$ reference electrode (Thermo Orion 900200, Beverly, MA, USA).

A pH meter GLP 22 (Crison Instruments, Barcelona, Spain) was used for $\mathrm{pH}$ measurements.

All measurements were carried out at room temperature $\left(20^{\circ} \mathrm{C}\right)$.

\subsection{Procedures}

\section{Experimental}


Graphite epoxy composite electrodes (GECs) were fabricated by using a PVC tube body $(6 \mathrm{~mm} \mathrm{i.} \mathrm{d.)} \mathrm{and} \mathrm{a}$ small copper disk soldered at the end of an electrical connector. The working surface is an epoxy-graphite conductive composite, formed by a mixture of $20 \%$ graphite powder (Merck, Darmstadt, Germany) and $80 \%$ of epoxy resin, Epotek H77, and its corresponding hardener (both from Epoxy Technology, Billerica, MA, USA), deposited on the cavity of the plastic body [19]. The composite material was cured at $80{ }^{\circ} \mathrm{C}$ for 3 days. Prior to their functionalization, the electrode surface was moistened with MilliQ water and then polished on abrasive sandpaper (400, 600, 800, 1000, and 1200 grit) and finally on alumina polishing strips (301044-001, Orion) in order to obtain a reproducible electrochemical surface.

\subsubsection{Preparation of modified GECs}

The specific steps for the modification of the GEC are described below [20].

-Diazonium salt electrochemical grafting: The in situ generation of the aryl diazonium was performed by adding $5 \cdot 10^{-3}$ equivalents of sodium nitrite to an acidic solution (1 $\mathrm{M}$ aqueous $\mathrm{HCl}$ ) of $\mathrm{ABA}$. These solutions were mixed for about $30 \mathrm{~min}$ in an ice bath, prior to the electrochemical grafting process [21] conducted by scanning the potential at $0.2 \mathrm{~V} \mathrm{~s}^{-1}$ from $0 \mathrm{~V}$ to $-1 \mathrm{~V}$ for 100 cycles. The functionalized electrodes were thoroughly rinsed with Milli-Q water and methanol to remove any physisorbed compounds.

-Covalent immobilization of crown ethers via carbodiimide coupling: The carboxyl groups of the electrografted diazonium salt were activated by incubating the functionalized electrodes in a $26 \mathrm{mM}$ EDC and $35 \mathrm{mM}$ sulfo-NHS solution in $100 \mathrm{mM}$ MES buffer ( $\mathrm{pH} 4.5)$ for 1 h. In order to conjugate the carboxy-functionalized electrode with the carboxy-modified ligands, a lysine spacer was intercalated in between, by using its two amino functionalities to form amido bonds [6]. The surface activated groups reacted overnight with the $\alpha$-amine group of the lysine at $4{ }^{\circ} \mathrm{C}$. Prior to cross linking with EDC/sulfoNHS, $2.9 \mathrm{mg}$ of 4-carboxybenzo-18-crown-6 or 4carboxybenzo-15-crown-5 were incubated with $100 \mu \mathrm{L} 5$ $\mathrm{mM}$ lysine in $0.1 \mathrm{M}$ MES buffer for $3 \mathrm{~h}$.

Figure 1 illustrates the CB-15-crown-5-GEC and CB-18crown-6-GEC electrodes modified by electrochemical grafting.

The electrochemical response using $2 \mathrm{mM}$ ferrocyanide/ferricyanide as redox probe in $100 \mathrm{mM}$ phosphate buffer ( $\mathrm{pH}$ 7.4) was investigated at each functionalization step using cyclic voltammetry (CV) leading voltammograms that confirm the modifications occurring on the electrode surface (Figure not shown). This procedure has been tested [6] with a high repeatability and a noticeable reproducibility.

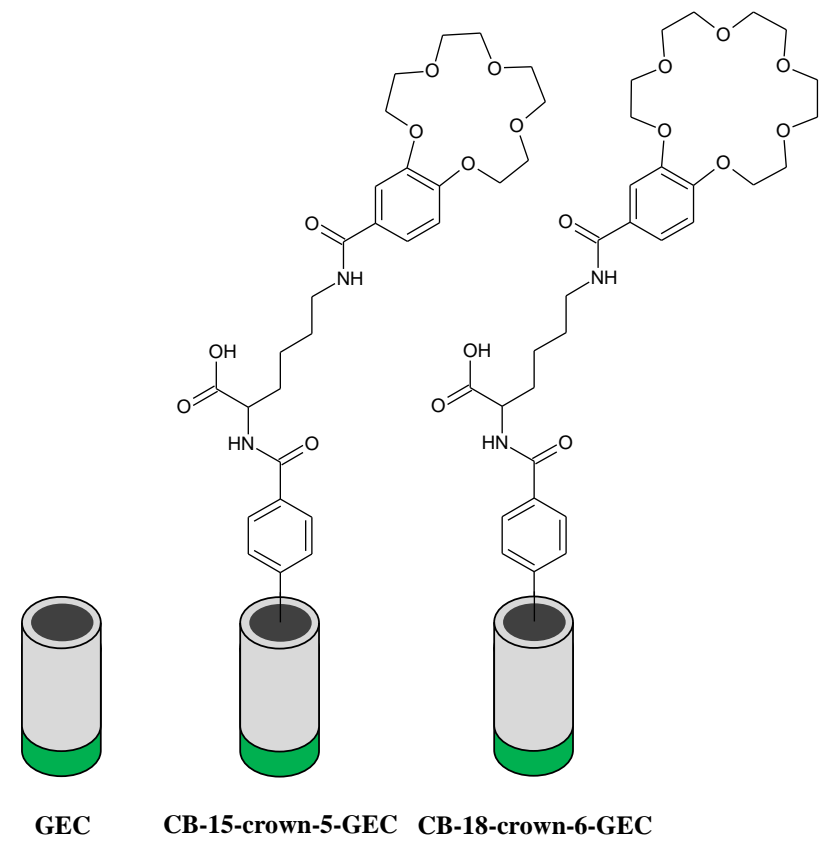

Fig. 1. Scheme of the three-sensor array: GEC, CB-15-crown-5GEC and CB-18-crown-6-GEC.

\subsubsection{Voltammetric measurements}

Before each set of measurements, the electrodes were scanned in acetic acid/acetate buffer solution in order to get stable voltammetric responses.

Voltammetric determinations using GEC, CB-18-crown6-GEC and CB-15-crown-5-GEC of $\mathrm{Cd}(\mathrm{II}), \mathrm{Pb}(\mathrm{II})$ and $\mathrm{Hg}(\mathrm{II})$, were done, without the need of any oxygen removal, at a deposition potential $\left(\mathrm{E}_{\mathrm{d}}\right)$ of $-1.4 \mathrm{~V}$, applied with stirring during a deposition time $\left(\mathrm{t}_{\mathrm{d}}\right)$ of $300 \mathrm{~s}$ and followed for a rest period $\left(\mathrm{t}_{\mathrm{r}}\right)$ of $10 \mathrm{~s}$. Determinations were done by scanning potential from -1.4 to $+0.7 \mathrm{~V}$ using a step potential of $4 \mathrm{mV}$ and pulse amplitude of $50 \mathrm{mV}$. Calibration plots were obtained by increasing metal concentrations in $\mathrm{pH} 4.5$ acetic acid/acetate buffer media.

In order to eliminate any remaining bound metals from the electrode, an electrochemical cleaning stage was considered between measurements. This stage was performed by applying a conditioning potential $\left(\mathrm{E}_{\mathrm{cond}}\right)$ of $1.20 \mathrm{~V}$ for $240 \mathrm{~s}$ after each measurement, in a cell containing fresh buffer solution.

To allow the multimetal simultaneous determination, a response model was built using artificial neural networks (ANN). For this aim, voltammetric scans of a total set of 
37 multimetal mixed samples in the concentration range 0 - $200 \mu \mathrm{g} \cdot \mathrm{L}^{-1}$ were recorded at the same experimental conditions as calibration plots. The set of samples was divided into two data subsets: a training subset formed by 27 samples $(72.9 \%)$, which were distributed in a factorial design [22] with 3 factors and 3 levels, and used to establish the response model and the testing subset formed by 10 samples $(27.1 \%)$, randomly distributed along the experimental domain, used to evaluate the model predictive response; additionally 3 certified samples, also containing the three metals randomly distributed along the experimental domain, were used to evaluate the applicability of the three-sensor array for the simultaneous determination of $\mathrm{Pb}(\mathrm{II}), \mathrm{Cd}(\mathrm{II})$ and $\mathrm{Hg}(\mathrm{II})$ in real samples.

Training and testing subset samples were manually prepared by appropriate dilution from the prepared metal stock solutions $10^{-2} \mathrm{~mol} \cdot \mathrm{L}^{-1}$ in $\mathrm{pH} 4.5$ acetic acid/acetate buffer, whereas certified samples were manually prepared by appropriate dilution from $1000 \mathrm{mg} \cdot \mathrm{L}^{-1}$ certified standard solutions in $\mathrm{pH} 4.5$ acetic acid/acetate buffer.

All experiments were carried out without any oxygen removal.

\subsubsection{Data processing}

In order to reduce the large amount of information generated for each sample (3 sensors x 431 current values at different potential) and its multiway nature a preprocessing stage was necessary to compress the original data. The objective of this step is to reduce the complexity of the input data while preserving the relevant information; also the compression of the data allows to reduce the training time, avoid redundancy in input data and to obtain a model with better generalization ability.

The chosen method was the Discrete Wavelet Transform (DWT) [23], each voltammogram was compressed using Daubechies 3 wavelet mother function and a 4 decomposition level. In this manner, the original data was reduced to 93 coefficients without losing relevant information, achieving a compression ratio up to $92.3 \%$.

Chemometric processing of data was performed by specific routines written by the authors using MATLAB 8.4 (MathWorks, Natick, MA) and its Neural Network Toolbox (v.8.2.1).

\section{Results and discussion}

As the most novel part of the present work is the part concerning $\mathrm{Hg}$ (II) sensing, this was studied in detail; next, the sensor array was employed for the simultaneous determination of the three considered metals, in an electronic tongue approach.

\subsection{Calibration data}

Most influential parameters in the ASV voltammetric response for a given metal are the operating parameters, such as deposition potential $\left(\mathrm{E}_{\mathrm{d}}\right)$, the accumulation potential $\left(\mathrm{t}_{\mathrm{d}}\right)$ and $\mathrm{pH}$ of the medium. The optimized compromise conditions for the simultaneous determination of $\mathrm{Pb}$ (II), $\mathrm{Cd}(\mathrm{II})$ and $\mathrm{Hg}$ (II) using the threesensor array were an $E_{d}$ of $-1.4 \mathrm{~V}$ with stirring during a $t_{d}$ of $300 \mathrm{~s}$ and followed by a rest period of $10 \mathrm{~s}$ at the same applied potential in $0.1 \mathrm{~mol} \mathrm{L^{-1 }}$ acetic acid/acetate buffer $\mathrm{pH} 4.5$.

Once stablished the working conditions, the electrodes of the array (GEC, CB-18-crown-6-GEC and CB-15crown-5-GEC) were analytically characterized for the determination of $\mathrm{Hg}$ (II) given there are no previous studies in this regard; on the other hand, responses towards $\mathrm{Cd}(\mathrm{II})$ and $\mathrm{Pb}$ (II) were already studied in a related work, involving the triad of metals $\mathrm{Cd}(\mathrm{II}), \mathrm{Pb}$ (II) and $\mathrm{Cu}$ (II) [6].

First of all, individual calibration of $\mathrm{Hg}$ (II) ion by stripping voltammetry (DPASV) was carried out using the three-sensor array. The LOD was calculated as 3 times the standard deviation of the intercept over the slope of the calibration curve of the target ions. LOQ was evaluated by considering 10 times the previous ratio. The lowest value of the linear concentration range was established from the corresponding limit of quantification (LOQ). For LOD and LOQ determinations, eleven different standards of the considered ion were used to build the calibration lines.

The three sensors evaluated provided a well-defined stripping peak over the considered concentration range. Excellent linear responses of peak currents versus $\mathrm{Hg}$ (II) concentrations was obtained for GEC, CB-18-crown-6GEC and CB-15-crown-5-GEC up to a maximum concentration level of $200 \mu \mathrm{g} \mathrm{L}^{-1}$. The corresponding

Table 1. Calibration data for the determination of $\mathrm{Hg}$ (II) on GEC, CB-18-crown-6-GEC and CB-15-crown-5-GEC at Ed of -1.4 V using a $t_{d}$ of $120 \mathrm{~s}$ at $\mathrm{pH} 4.5$. 


\begin{tabular}{llll}
\cline { 2 - 4 } & \multicolumn{1}{c}{ GEC } & CB-18-crown-6-GEC & CB-15-crown-5-GEC \\
\hline Regression $^{\mathbf{a}}$ & $\mathrm{y}=0.157 \mathrm{x}+2.88$ & $\mathrm{y}=0.146+3.26$ & $\mathrm{y}=0.128+4.76$ \\
$\mathbf{R}^{2}$ & 0.985 & 0.995 & 0.995 \\
Linear range $\left(\boldsymbol{\mu g} \mathbf{~ L}^{-\mathbf{1}}\right)^{\mathbf{b}}$ & $37-200$ & $43-200$ & $40-200$ \\
$\mathbf{L O D}\left(\boldsymbol{\mu g} \mathbf{~ L}^{-\mathbf{1}}\right)$ & 11 & 13 & 12 \\
\hline
\end{tabular}

${ }^{a} \mathrm{y}$ is the peak height $(\mu \mathrm{A})$ and $\mathrm{x}$ the concentration $\left(\mu \mathrm{g} \mathrm{L^{-1 }}\right)$.

${ }^{b}$ The lowest value of the linear range was considered from the LOQ.

regression equations and the correlation coefficient for the sensors of the array are shown in Table 1.

With respect to the sensitivities considered as the value obtained from the slope of the calibration curves, it can be stated that: $\mathrm{Hg}(\mathrm{II})$ shows a very similar sensitivity for the three considered sensors suggesting that the three electrodes respond in the same way versus $\mathrm{Hg}$ (II). However, comparing the two crown ether - modified sensors it can be observed that their sensitivities are slightly different suggesting that CB-18-crown-6-GEC offers a higher interaction than CB-15-crown-5-GEC with $\mathrm{Hg}$ (II). Related to the unmodified GEC it has a sensitivity slightly higher than other sensors, this fact is because the graphite structure where the mercury can be introduced into the own structure of the graphite making it especially sensitive. As shown in Table 1, both LOD and LOQ were at the level of $\mu \mathrm{g} \mathrm{L}^{-1}$ for all considered sensors. In comparison with previous results achieved at other crown ether - modified electrodes, the LODs provided by CB-18crown-6-GEC and CB-15-crown-5-GEC sensors were similar than the LOD obtained using a ferrocenoylpolythia crown ether-Nafion-modified glassy carbon electrode [13], and much lower than the LOD achieved using a carbon-paste electrodes modified with 18-crown-6 [12]. It must be point out that in this work an enrichment time of $300 \mathrm{~s}$ was selected looking for a compromise between the peak currents and the time of the analysis, nevertheless lower concentrations ranges and better detection limits could easily be achieved using the proposed method by increasing the enrichment time.

Comparing both unmodified GEC and crown ether modified GEC it can be observed that the LOD and LOQ values obtained for both CB-18-crown-6-GEC and CB-15crown-5-GEC sensors are in the range of those obtained for the unmodified graphite composite electrode.
Therefore the reported calibration data suggest that all considered sensors could be fully suitable for the determination of $\mathrm{Hg}(\mathrm{II})$ at low $\mu \mathrm{g} \cdot \mathrm{L}^{-1}$ level in natural samples. However, until no simultaneous determinations of $\mathrm{Hg}$ (II) with other heavy metal ions such as $\mathrm{Cd}(\mathrm{II})$ and $\mathrm{Pb}$ (II) are performed, it cannot be established which of the considered sensor is the best especially in terms of selectivity for $\mathrm{Hg}$ (II) determination.

\subsection{Multimetal Stripping Voltammetric Measurements}

Before the application of the three considered sensors as an array for the simultaneous determination of $\mathrm{Cd}(\mathrm{II})$, $\mathrm{Pb}$ (II) and $\mathrm{Hg}$ (II) is import to know if this array can add some discrimination power to resolve the mixture. Therefore, the cross-response of these sensors was examined.

Considering that the immobilized crown ethers on the GEC surface are used as molecular collector with ability to selectively coordinate with the metal ions, both the ionic diameter of metal ions and the cavity size of the crown ethers play a crucial role for the complex formation by means of ion-dipole interaction with metal ions. Although, it is known that both CB-18-crown-6 and CB-15-crown-5 exhibit a cross-response for $\mathrm{Cd}$ (II) and $\mathrm{Pb}$ (II) [6], it is unknown whether with $\mathrm{Hg}$ (II) has this characteristic response. With this aim voltammetric peak current responses for equal concentrations of metal ion solution (175 $\mu \mathrm{g} \mathrm{L}^{-1}$ of $\mathrm{Cd}(\mathrm{II}), \mathrm{Pb}(\mathrm{II})$ and $\mathrm{Hg}(\mathrm{II})$ ) were recorded using the three-sensor array. Figure 2 shows that, on the one hand, CB-18-crown-6-GEC and GEC exhibit the highest interaction with $\mathrm{Pb}$ (II) and $\mathrm{Hg}$ (II) being the iondipole interaction of CB-18-crown-6-GEC with $\mathrm{Hg}$ (II) slightly higher than with $\mathrm{Pb}(\mathrm{II})$; on the other hand, $\mathrm{CB}-15$ - 
crown-5-GEC offers the highest selective complex forming ability with $\mathrm{Hg}$ (II) followed by $\mathrm{Cd}(\mathrm{II})$. Although, for the three considered sensors $\mathrm{Hg}$ (II) shows a similar interaction, it can be seen that CB-18-crown-6-GEC is a little bit more selective than CB-15-crown-5-GEC. Comparing the ionic diameter of the considered metal ions (1.9 $\AA$ for $\mathrm{Cd}$ (II), $2.1 \AA$ for $\mathrm{Hg}$ (II), and $2.4 \AA$ for $\mathrm{Pb}$ (II)) with the cavity size of both crown ethers (cavity diameter, 1.7-2.2 $\AA$ for CB-15-crown-5 and 2.6-3.2 $\AA$ for CB-18crown-6), the described behavior is consistent with studies by Christensen et al. in 1971 who suggested that cation diameter to host cavity size ratios of $0.75-0.90$ are favorable for direct ion-crown ether binding [24]. Thus, ratios of $0.66-0.81$ and $0.75-0.92$ for $\mathrm{Hg}(\mathrm{II})$ and $\mathrm{Pb}(\mathrm{II})$, respectively, were achieved for $\mathrm{CB}-18$-crown-6 reflecting the size match for those ions. Likewise, a ratio of 0.86-1.11 for CB-15-crown-5 were obtained for $\mathrm{Cd}(\mathrm{II})$ consistent with the observed selectivities.

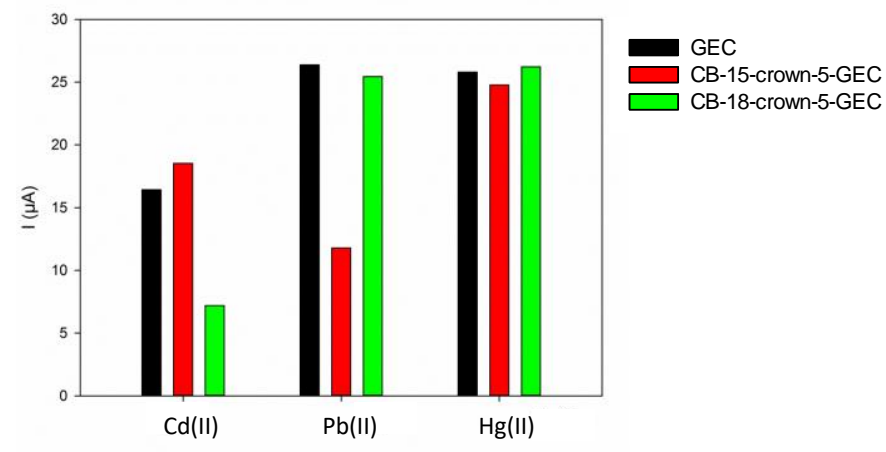

Fig. 2. Differential Pulse anodic stripping voltammetric sensitivity, from peak current of $175 \mu \mathrm{g} \mathrm{L}^{-1}$ of $\mathrm{Cd}(\mathrm{II}), \mathrm{Pb}(\mathrm{II})$ and $\mathrm{Hg}$ (II) using the three-sensor array. Black: graphite epoxy composite electrode (GEC); red: CB-15crown-5-GEC; green: CB-18-crown-6-GEC.

Therefore, from Figure 2 it can be evidenced the existence of cross-response between the three metal ions and the considered sensors. In this way, whereas additionally a maximum signal for each metal is obtained for a different electrode, the use of the three-sensor array would provide higher information to resolve the multimetal mixture than that obtained from a single electrode. As an example, a sample of four stripping voltammograms obtained using CB-18-crown-6-GEC (arbitrary concentrations) is displayed in Figure 3. Relatively well-defined stripping peaks without any clear evidence of signal splitting or overlapping effect were observed over the considered concentration range. The potential of the oxidation peak of each considered metal in the complex voltammograms was assigned at $c a .-0.73 \mathrm{~V}$,
$-0.48 \mathrm{~V}$, and $0.31 \mathrm{~V}$ for $\mathrm{Cd}(\mathrm{II}), \mathrm{Pb}(\mathrm{II})$ and $\mathrm{Hg}(\mathrm{II})$ respectively. A comparison between the voltammograms provided by unmodified GEC and both crown ethersmodified sensors displayed no significant differences in metal peak shapes and peak potentials (at shown concentration levels), however different degree of metal interactions were observed in agreement with metal complex selectivity (inset in Figure 3). Thus, the stripping voltammetric response will be different depending on the metal ion concentration in each calibration mixture, the used sensor and the metal complex selectivity.

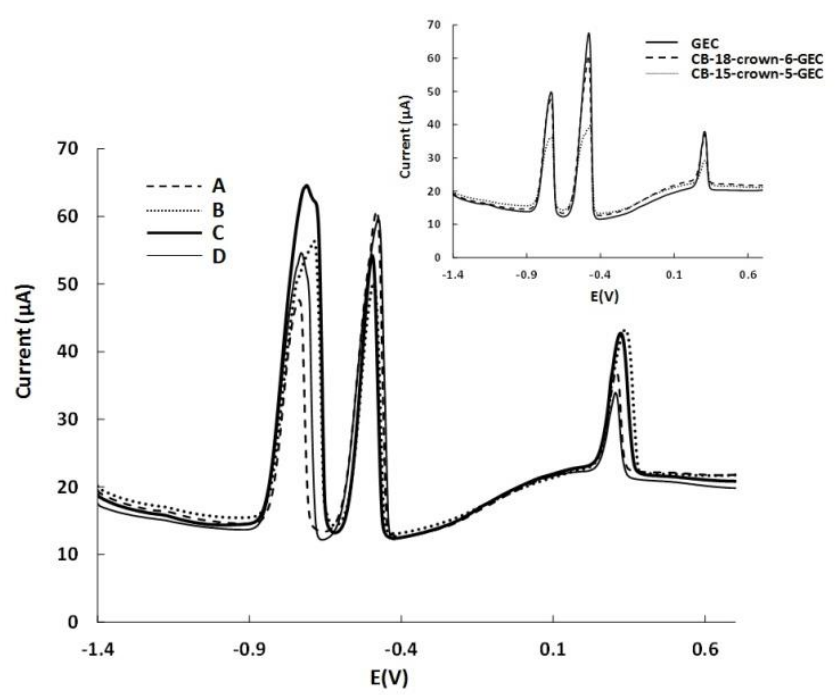

Fig. 3. Some differential pulse anodic stripping voltammograms generated during the building of the response model recorded on a CB-18-crown-6-GEC sensor at $\mathrm{pH} 4.5$ using a $\mathrm{E}_{\mathrm{d}}$ of $-1.40 \mathrm{~V}$ during $300 \mathrm{~s}$ and $\mathrm{t}_{\mathrm{r}}$ of $10 \mathrm{~s}$. Sample composition: (A) $31.1 \mu \mathrm{g} \mathrm{L}^{-1}$ of $\mathrm{Cd}(\mathrm{II}), 147.1 \mu \mathrm{g} \mathrm{L} \mathrm{L}^{-1}$ of $\mathrm{Pb}$ (II) and $27.5 \mu \mathrm{g} \mathrm{\textrm {L } ^ { - 1 }}$ of $\mathrm{Hg}$ (II); (B) $124.9 \mu \mathrm{g} \mathrm{L}^{-1}$ of $\mathrm{Cd}(\mathrm{II}), 92.5 \mu \mathrm{g} \mathrm{L} \mathrm{L}^{-1}$ of $\mathrm{Pb}(\mathrm{II})$ and $136.2 \mu \mathrm{g} \mathrm{L}^{-1}$ of $\mathrm{Hg}(\mathrm{II})$; (C) $177.4 \mu \mathrm{g} \mathrm{L}^{-1}$ of Cd(II), $70.0 \mu \mathrm{g} \mathrm{L}^{-1}$ of $\mathrm{Pb}$ (II) and 80.1 $\mu \mathrm{g} \mathrm{L}^{-1}$ of $\mathrm{Hg}$ (II); (D) $94.2 \mu \mathrm{g} \mathrm{L}^{-1}$ of Cd(II), $162.9 \mu \mathrm{g} \mathrm{L}^{-1}$ of $\mathrm{Pb}$ (II) and $7.5 \mu \mathrm{g} \mathrm{L}^{-1}$ of $\mathrm{Hg}(\mathrm{II})$. Inset: comparison between the response of the three-sensor array for sample composition (A).

As the next step, the sets of voltammograms of heavy metal mixtures obtained from the three-sensor array were postulated to be used to calibrate $\mathrm{Cd}$ (II), $\mathrm{Pb}$ (II) and $\mathrm{Hg}$ (II) using an appropriate ANN model that may consider any non-linearity or splitting in the determination of the considered metal ions.

\subsection{Quantification of the metal mixtures}

Once the data were compressed by use of Wavelet Transform in this study case, the first step in building the appropriate ANN model is choosing the topology of the 
Table 2. Results of the fitted regression lines for the comparison between obtained vs. expected values, for the training and testing subsets of samples and the different metal species (intervals calculated at the $95 \%$ confidence level).

\begin{tabular}{|c|c|c|c|c|c|c|c|c|}
\hline & Metal & Correlation & Slope & Intercept (ppb) & $R M S E(p p b)$ & NRMSE & Total RMSE (ppb) & Total NRMSE \\
\hline \multirow{3}{*}{ train subset } & $\mathrm{Hg}(\mathrm{II})$ & 0.999 & $0.99 \pm 0.04$ & $0.74 \pm 4.91$ & 3.89 & 0.019 & \multirow{3}{*}{3.69} & \multirow{3}{*}{0.018} \\
\hline & $\mathrm{Pb}(\mathrm{II})$ & 0.999 & $0.99 \pm 0.02$ & $0.49 \pm 2.46$ & 1.97 & 0.010 & & \\
\hline & $\mathrm{Cd}(\mathrm{II})$ & 0.998 & $0.99 \pm 0.05$ & $0.71 \pm 5.95$ & 4.69 & 0.023 & & \\
\hline \multirow{3}{*}{ test subset } & $\mathrm{Hg}(\mathrm{II})$ & 0.982 & $1.25 \pm 0.39$ & $-14.26 \pm 40.44$ & 21.71 & 0.109 & \multirow{3}{*}{19.35} & \multirow{3}{*}{0.096} \\
\hline & $\mathrm{Pb}(\mathrm{II})$ & 0.986 & $0.94 \pm 0.25$ & $16.11 \pm 28.66$ & 14.10 & 0.071 & & \\
\hline & $\mathrm{Cd}(\mathrm{II})$ & 0.927 & $0.77 \pm 0.51$ & $35.24 \pm 58.97$ & 23.17 & 0.116 & & \\
\hline
\end{tabular}

RMSE: root mean square error; NRMSE: normalized root mean square error

neural network used. Normally, given the difficulties to predict the optimal settings in advance this is a trial-anderror process, where several parameters (training algorithms, number of hidden layers, transfer functions, etc.) are fine-tuned in order to find the best configuration that optimizes the performance of the model [25].

In consequence, the samples from the training subset were used for building the ANN model, and its accuracy was then evaluated towards samples of the external test subset by employing the developed model to predict the concentrations of the metals of those samples (external validation). Taking into account that the external test subset data is not used at all for the modelling, its goodness of fit is a measure of the completed modelling performance.

Model prediction abilities are shown in the comparison graphs (obtained vs. expected concentrations) for all ions, both for training subset and testing subsets. The factors considered for the selection of the best model were the accuracy of fit, evaluated as the smaller RMSE (root mean squared error) [26] and additionally, regression parameters from the comparison graphs close to the ideal values (i.e. slope and correlation coefficient equal 1 , and intercept equal 0 ), meaning that there are no significant differences between the values predicted by the ANN model and those expected and provided by the reference method.

In our case, the resolution of the $\mathrm{Cd}$ (II), $\mathrm{Pb}$ (II) and $\mathrm{Hg}$ (II) mixtures was attempted using the data from the three voltammetric sensor array. To this aim, the set of samples was measured with the three electrodes (unmodified GEC, CB-15-crown-5-GEC and CB-18-crown-6-GEC) and the obtained voltammetric responses were compressed employing DWT and the different ANN models were optimized. After a systematic study optimizing the different parameters, the final architecture of the ANN model had 93 neurons in the input layer, 4 neurons and satlins transfer function in the hidden layer

and 3 neurons and purelin transfer function in the output layer, providing the concentrations of the three species considered. Figure 4 illustrates the comparison graphs of predicted $v s$. expected concentrations for the considered compounds for training ( $\bullet$, solid line) and testing subsets ( $\circ$, dashed line). As it can be observed, a satisfactory trend was obtained for all three metal ions with regression lines close to the theoretical ones (long dashed line). Table 2 shows the calculated linear comparison parameters being near the ideal value, with correlation coefficients and slopes with values very close to 1 and intercepts quite close to 0 .

Additionally, to assess the applicability of the threesensor array to real samples, an attempt was performed to simultaneously determine $\mathrm{Cd}$ (II), $\mathrm{Pb}$ (II) and $\mathrm{Hg}$ (II) in 3 certified samples, randomly distributed along the experimental domain. Figure 4 ( $\vec{*}$ ) shows the linear regression results for $\mathrm{Cd}(\mathrm{II}), \mathrm{Pb}(\mathrm{II})$ and $\mathrm{Hg}(\mathrm{II})$. The general trend is quite satisfactory for all the considered metal ions with slopes and intercepts close to 1 and 0 respectively, and with correlations being also significant (Table 2). Although, $\mathrm{Cd}$ (II) is the analyte with the worst performance especially with a somewhat lesser correlation and higher confidence interval of the intercept value. 
a)

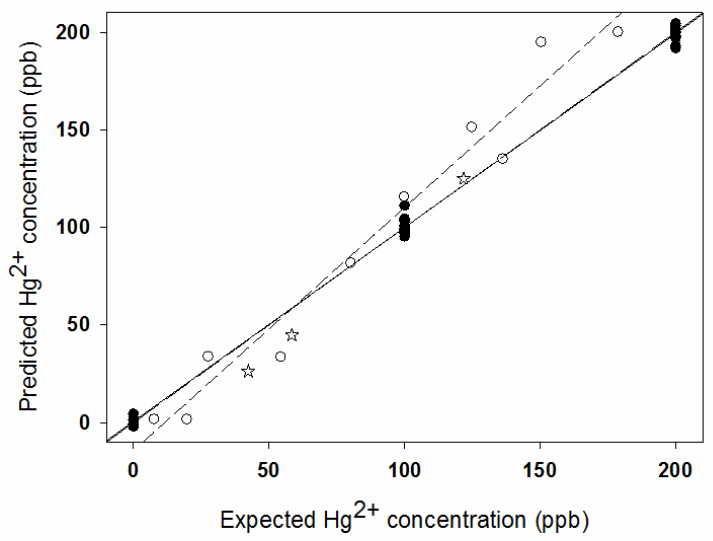

b)

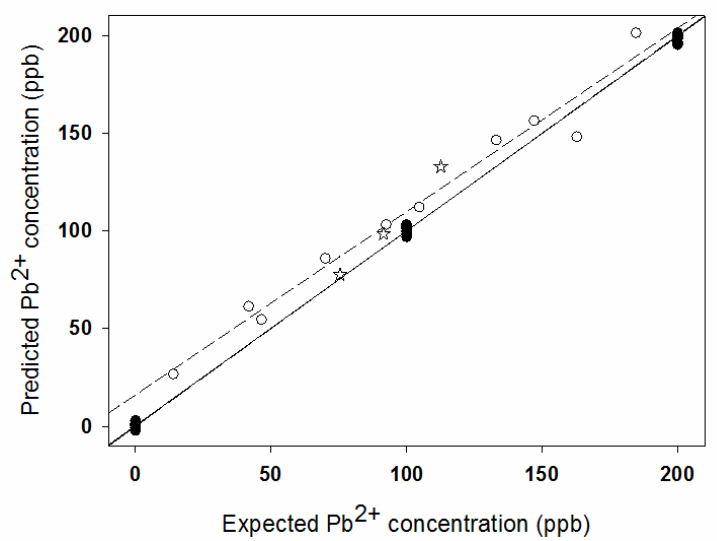

c)

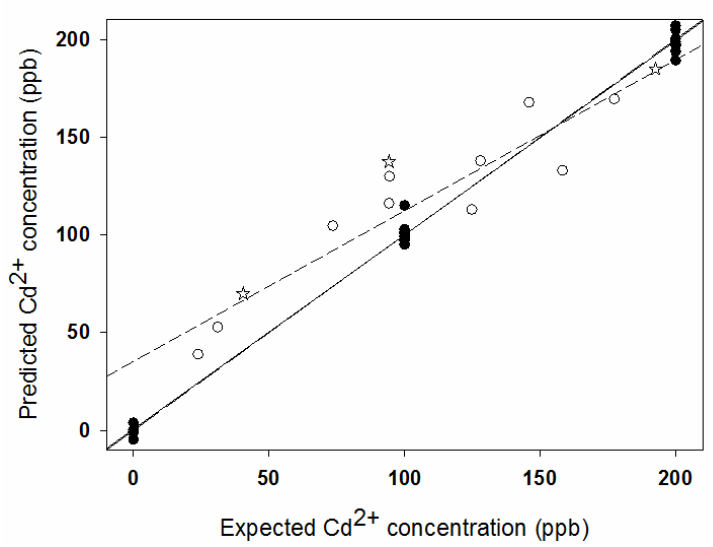

Fig. 4. Modelling ability of the optimized ANN for the three-sensor array. Sets adjustments of obtained vs. expected concentrations for (a) $\mathrm{Cd}$ (II), (b) $\mathrm{Pb}$ (II) and (c) $\mathrm{Hg}$ (II), for training subset $(\bullet$, solid line), testing subsets $(\circ$,dashed line) and certified samples ( $\vec{k})$. Long dashed line corresponds to theoretical diagonal line.

\section{Conclusions}

In this work, a three-sensor array constituted by an unmodified GEC and two GECs modified with CB-18crown-6 and CB-15-crown-5 respectively, which were immobilized on aryl diazonium salt monolayers anchored to the electrode surface, was successfully applied for the simultaneous determination of $\mathrm{Cd}(\mathrm{II}), \mathrm{Pb}$ (II) and $\mathrm{Hg}$ (II) by DPASV. The three-sensor array was firstly analytical studied for the determination of $\mathrm{Hg}$ (II) providing a wellshaped stripping peak over the considered concentration range. The achieved LODs and LOQs were at levels of $\mu \mathrm{g}$ $\mathrm{L}^{-1}$, which are similar or even much lower to those obtained for the determination of $\mathrm{Hg}$ (II) with other crown ether - modified electrodes [12-13].

Taking advantage of the crown ethers complex forming ability with the considered metal ions, the use of the threesensor array allows the existence of a cross-response between the three metal ions and the considered sensors adding the discrimination power to resolve the $\mathrm{Cd}(\mathrm{II})$, $\mathrm{Pb}$ (II) and $\mathrm{Hg}$ (II) mixture.

In this sense, in this work, the simultaneous quantification of $\mathrm{Cd}$ (II), $\mathrm{Pb}$ (II) and $\mathrm{Hg}$ (II) was satisfactory achieved by combining the set of voltammetric measurements with chemometric tools. Thus, voltammetric data obtained using the three-sensor array was preprocessed by DWT and coupled with an artificial neural network. The experiments exhibited similar performance in all training and testing correlation coefficients, obtained from the predicted $v s$. expected concentrations comparison graphs, which were in all cases higher than 0.927. Under the above mentioned conditions, the simultaneous determination of $\mathrm{Cd}(\mathrm{II}), \mathrm{Pb}$ (II) and $\mathrm{Hg}(\mathrm{II})$ in certified samples using the three-sensor array was successfully attempted obtaining a satisfactory trend for the three considered metal ions with correlation coefficients of 0.998 and 0.999 for $\mathrm{Pb}$ (II) and $\mathrm{Hg}$ (II), respectively, whereas for $\mathrm{Cd}(\mathrm{II})$ a lower correlation (0.964) and a higher confidence interval of the intercept value are achieved, the recovery values were $80.7 \%$, $109.4 \%$ and $138.1 \%$ for $\mathrm{Hg}(\mathrm{II}), \mathrm{Pb}$ (II) and $\mathrm{Cd}(\mathrm{II})$ respectively. In similar works performed, $\mathrm{Cd}(\mathrm{II})$ has been always the worst performing metals probably because of non-linearities in measured signals originated at intermetallic reduced compounds $[6,27]$.

At the sight of the satisfactory results reached in this work, the proposed methodology seems to be perfectly suitable for the determination of heavy metals ions in environmental and biological samples at the ultra-trace level. Even so, further research focused on the application of the proposed method for drinking water analysis is required taking into consideration the guidelines for drinking water quality [28]; since $\mathrm{Cd}$ (II) and $\mathrm{Pb}$ (II) could be successfully determined according to the proposed experimental conditions [6], whereas for $\mathrm{Hg}($ II) determination an increase of the deposition time is required. 


\section{Acknowledgements}

The authors acknowledge financial support from the Spanish Ministry of Science and Innovation (MINECO, through projects CTQ2013-41577-P and CTQ201232863). M del Valle acknowledges support by the Catalonia program ICREA Academia.

\section{References}

[1] E. Lawrence, A.R.W. Jackson, J.M. Jackson, Longman Dictionary of Environmental Science, Addison Wesley Longman, Harlow, UK, 1998.

[2] L. Järup, British Medical Bulletin 2003, 68, 167-182.

[3] J. Wang, Stripping Analysis: Principles, Instrumentation and Applications, VCH, Deerfield Beach, FL 1985.

[4] J. Gooding, D. Hibbert, W. Yang, Sensors 2001, 1, 75-90.

[5] N. Serrano, B. Prieto-Simón, X. Cetó, M. del Valle, Talanta 2014, 125, 159-166.

[6] N. Serrano, A. González-Calabuig, M. del Valle, Talanta 2015, 138, 130-137.

[7] C. Pérez-Ràfols, N. Serrano, J.M. Díaz-Cruz, C. Ariño, M. Esteban, Talanta 2015, 144, 569-573.

[8] J. W. Steed, J. L. Atwood, Supramolecular Chemistry, Wiley, Chichester, UK 2009.

[9]. S. V. Prabhu, R. P. Baldwin, L, Kryger, Electroanalysis 1989, $1,13-21$.

[10] S. Anandhakumar, J. Mathiyarasu, Microchim. Acta 2013, 180, 1065-1071.

[11] V. S. Ijeri, A. K. Srivastava, Anal. Sci. 2001, 17, 605-608.

[12] J. Wang, M. Bonakdar, Talanta 1988, 35, 277-280.

[13] G. Zhiqiang, L. Peibiao, Z. Zaofan, Microchem. J. 1991, 43, 121-132.

[14] S. Tanaka, H. Yoshida, Talanta 1989, 36, 1044-1046.

[15] S. Cheraghi, M. A. Taher, H. Fazelirad, Microchim. Acta 2013, 180, 1157-1163.

[16] M. V. Tsymbal, I. Ya. Tuijan, Z. A. Temerdashev, K. Z. Brainina, Electroanalysis 1994, 6, 113-117.

[17] V. S. Ijeri, A. K. Srivastava, Fresenius J. Anal. Chem. 2000, $367,373-377$.

[18] M. del Valle, Int. J. Electrochem. 2012, Volume 2012, Article ID 986025, 11 pages.

[19] A. Bonanni, M.J. Esplandiu, M.I. Pividori, S. Alegret, M. del Valle, Anal. Bioanal. Chem. 2006, 385, 1195-1201.

[20] C. Ocaña, M. del Valle, Microchim. Acta 2014, 181, 355363.

[21] D. Belanger, J. Pinson, Chem. Soc. Rev. 2011, 40, 39954048 .
[22] X. Cetó, F. Céspedes, M.I. Pividori, J.M. Gutiérrez, M. del Valle, Analyst 2012, 137 349-356.

[23] M. del Valle, R. Muñoz, J.M. Gutiérrez, Wavelets: Classification, Theory and Applications, Nova Science Pub Inc, New York, US, 2011.

[24] J. J. Christensen, J. O. Hill, R. M. Izatt, Science 1971, 174, 459-467.

[25] F. Despagne, D. L. Massart, Analyst 1998, 123, 157R-178R.

[26] X. Cetó, F. Céspedes, M. del Valle, Microchim. Acta, 2013, 180, 319-330.

[27] J. M. Gutierrez, L. Moreno-Baron, F. Cespedes, R. Muñoz, M. del Valle, Electroanalysis 2009, 21, 445-451.

[28] Guidelines for Drinking-water Quality - 4th ed, World Health Organization, Geneva, Switzerland, 2011. 